\title{
On modular inequalities of interval-valued fuzzy soft sets characterized by soft $J$-inclusions
}

\author{
Xiaoyan Liu' ${ }^{1}$, Feng Feng ${ }^{1 *}$, Ronald R Yager ${ }^{2}$, Bijan Davvaz ${ }^{3}$ and Madad Khan ${ }^{4,5}$
}

${ }^{*}$ Correspondence:
fengnix@hotmail.com
'Department of Applied
Mathematics, School of Science,
Xi'an University of Posts and
Telecommunications, Xi'an, 710121,
China
Full list of author information is
available at the end of the article

available at the end of the article

\begin{abstract}
This study aims to explore modular inequalities of interval-valued fuzzy soft sets characterized by Jun's soft J-inclusions. Using soft product operations of interval-valued fuzzy soft sets, we first investigate some basic properties of soft $J$-inclusions and soft $L$-inclusions. Then a new concept called upward directed interval-valued fuzzy soft sets is defined and some equivalent characterizations are presented. Furthermore, we consider modular laws in lattice theory and find that classical modular inequalities in lattice theory are not valid for interval-valued fuzzy soft sets. Finally, we present some interesting inequalities of interval-valued fuzzy soft sets by virtue of soft $J$-inclusions and related notions.
\end{abstract}

MSC: $03 \mathrm{E} 72$

Keywords: interval-valued fuzzy soft sets; interval-valued fuzzy sets; soft product operations; soft inclusions; modular inequalities

\section{Introduction}

It is worth noting that uncertainty arise from various domains has different nature and cannot be captured within a single mathematical framework. In addition to probability theory and statistics, we currently have some advanced soft computing methods such as fuzzy sets [1], rough sets [2], and also soft sets [3]. Molodtsov's soft set theory provides a relatively new mathematical approach to dealing with uncertainty from a parameterization point of view. In the past decades, a rapid development in this theory and various applications have been witnessed [4-24].

Some researchers endeavored to enrich soft sets by combining them with other soft computing models such as rough sets and fuzzy sets. Using soft sets as the granulation structures, Feng et al. [21] initiated soft approximation spaces and soft rough sets, which generalize Pawlak's rough sets based on soft sets. On the other hand, Maji et al. [25] initiated the study on hybrid structures involving both fuzzy sets and soft sets. They introduced the notion of fuzzy soft sets, which can be seen as a fuzzy generalization of Molodtsov's soft sets. Furthermore, Yang et al. [26] introduced interval-valued fuzzy soft sets which realize a common extension of both Molodtsov's soft sets and interval-valued fuzzy sets. It should be noted that there are several different kinds of soft inclusions (also known as soft subsets) in the literature [22, 23, 27, 28]. Feng and Li [29] investigated different types of soft subsets and the related soft equal relations in a systematic way. 
They also considered some interesting algebraic properties of soft product operations for Molodtsov's soft sets. Liu et al. [30] investigated these issues in the more general setting of interval-valued fuzzy soft sets. They revealed some non-classical algebraic properties of interval-valued fuzzy soft sets with respect to soft product operations, which are distinct from those of interval-valued fuzzy sets. As a continuation to this line of research, in the present paper we will focus on modular inequalities of interval-valued fuzzy soft sets characterized by Jun's soft $J$-inclusions.

The remainder of this study is organized as follows. Section 2 introduces some fundamental concepts and useful results regarding interval-valued fuzzy soft sets. Section 3 investigates some basic properties of Jun's soft $J$-inclusions and Liu's soft $L$-inclusions. In Section 4, we define upward directed interval-valued fuzzy soft sets and concentrate on discussing algebraic properties of soft product operations concerning idempotency. Section 5 is devoted to the exploration of modular inequalities of interval-valued fuzzy soft sets. Finally, the last section summarizes the study and suggests possible future works.

\section{Preliminaries}

Let $U$ be a universe and $E_{U}$ (or simply $E$ ) be the set of all parameters associated with objects in $U$, which is called a parameter space. We denote the power sets of $U$ by $\mathscr{P}(U)$. Then soft sets are defined as follows.

Definition 2.1 [3] A pair $\mathfrak{S}=(F, A)$ is called a soft set over $U$, where $A \subseteq E$ and $F: A \rightarrow$ $\mathscr{P}(U)$ is a set-valued mapping, called the approximate function of the soft set $\mathfrak{S}$.

By virtue of parametrization, a soft set could provide a series of approximate descriptions of a complicated object being perceived from various points of view. For any parameter $\epsilon \in A$, the subset $F(\epsilon) \subseteq U$ is called an $\epsilon$-approximate set, consisting of all $\epsilon$-approximate elements [3].

Next, let us consider the set $L^{I}=\{[x, y]: 0 \leq x \leq y \leq 1\}$ and the order relation $\leq_{L^{I}}$ given by

$$
\left[x_{1}, y_{1}\right] \leq_{L^{I}}\left[x_{2}, y_{2}\right] \quad \Leftrightarrow \quad x_{1} \leq x_{2}, y_{1} \leq y_{2}, \quad \forall\left[x_{1}, y_{1}\right],\left[x_{2}, y_{2}\right] \in L^{I}
$$

Then $\mathscr{L}^{I}=\left(L^{I}, \leq_{L^{I}}\right)$ is a complete lattice. An interval-valued fuzzy set on a universe $U$ is a mapping $\mu: U \rightarrow L^{I}$. The union, intersection and complement of interval-valued fuzzy sets can be obtained by canonically extending fuzzy set-theoretic operations to intervals. The set of all interval-valued fuzzy sets on $U$ is denoted by $\mathscr{I}(U)$.

Definition 2.2 [27] Let $(U, E)$ be a soft universe and $A \subseteq E$. A pair $\mathfrak{I}=(\widetilde{F}, A)$ is called an interval-valued fuzzy soft set over $U$, where $\widetilde{F}$ is a mapping given by $\widetilde{F}: A \rightarrow \mathscr{I}(U)$.

The mapping $\widetilde{F}: A \rightarrow \mathscr{I}(U)$ is also called the approximate function of the intervalvalued fuzzy soft set $\mathfrak{I}=(\widetilde{F}, A)$. The following two operations $\wedge$ and $\vee$ will be referred to as soft product operations of interval-valued fuzzy soft sets in general.

Definition 2.3 [26] Let $\mathfrak{A}=(\widetilde{F}, A)$ and $\mathfrak{B}=(\widetilde{G}, B)$ be two interval-valued fuzzy soft sets over $U$. The $\wedge$-product (also called AND operation) of interval-valued fuzzy soft sets $\mathfrak{A}$ and $\mathfrak{B}$ is an interval-valued fuzzy soft set defined by $\mathfrak{A} \wedge \mathfrak{B}=(H, A \times B)$, where $H(a, b)=$ $F(a) \cap G(b)$ for all $(a, b) \in A \times B$. 
Definition 2.4 [26] Let $\mathfrak{A}=(\widetilde{F}, A)$ and $\mathfrak{B}=(\widetilde{G}, B)$ be two interval-valued fuzzy soft sets over $U$. The $\vee$-product (also called OR operation) of interval-valued fuzzy soft sets $\mathfrak{A}$ and $\mathfrak{B}$ is an interval-valued fuzzy soft set defined by $\mathfrak{A} \vee \mathfrak{B}=(H, A \times B)$, where $H(a, b)=$ $F(a) \cup G(b)$ for all $(a, b) \in A \times B$.

We denote by $\mathscr{S}^{I}(U, E)$ the collection of all interval-valued fuzzy soft sets over $U$ with parameter space $E$. For more details on interval-valued fuzzy soft sets and related terminologies used below, we refer to the papers [26, 27, 31]. For convenience, we abbreviate the term 'interval-valued fuzzy' as IVF in what follows.

There are some different types of soft inclusion relations in the literature. Here we mainly introduce two of them, namely Jun's inclusion $\widetilde{\subseteq}_{J}$ in [27] and Liu's inclusion $\widetilde{\subseteq}_{L}$ in [28]. The readers who are interested in soft subsets and related topics are referred to papers [27-29].

Definition 2.5 [27] Let $(\widetilde{F}, A)$ and $(\widetilde{G}, B)$ be two IVF soft sets over $U$. Then $(\widetilde{F}, A)$ is called a IVF soft J-subset of $(\widetilde{G}, B)$, denoted $(\widetilde{F}, A) \widetilde{\subseteq}_{J}(\widetilde{G}, B)$, if for every $a \in A$ there exists $b \in B$ such that $\widetilde{F}(a) \subseteq \widetilde{G}(b)$. Two IVF soft sets $(\widetilde{F}, A)$ and $(\widetilde{G}, B)$ are said to be IVF soft J-equal, $\operatorname{denoted}(\widetilde{F}, A)=_{J}(\widetilde{G}, B)$, if $(\widetilde{F}, A) \widetilde{\subseteq}_{J}(\widetilde{G}, B)$ and $(\widetilde{G}, B) \widetilde{\subseteq}_{J}(\widetilde{F}, A)$.

Motivated by Jun and Yang's IVF soft $J$-subsets, Liu et al. [28] further introduced the following kinds of IVF soft subsets.

Definition 2.6 [28] Let $(\widetilde{F}, A)$ and $(\widetilde{G}, B)$ be two IVF soft sets over $U$. Then $(\widetilde{F}, A)$ is called a IVF soft $L$-subset of $(\widetilde{G}, B)$, denoted $(\widetilde{F}, A) \widetilde{\subseteq}_{L}(\widetilde{G}, B)$, if for every $a \in A$ there exists $b \in B$ such that $\widetilde{F}(a)=\widetilde{G}(b)$. Two IVF soft sets $(\widetilde{F}, A)$ and $(\widetilde{G}, B)$ are said to be IVF soft L-equal, denoted $(\widetilde{F}, A)={ }_{L}(\widetilde{G}, B)$, if $(\widetilde{F}, A) \widetilde{\subseteq}_{L}(\widetilde{G}, B)$ and $(\widetilde{G}, B) \widetilde{\subseteq}_{L}(\widetilde{F}, A)$.

Note that $\widetilde{\subseteq}_{J}$ and $\widetilde{\subseteq}_{L}$ are binary relations on $\mathscr{S}^{I}(U, E)$, which are called IVF soft Jinclusion and IVF soft L-inclusion, respectively. The following result is easily verified in virtue of the above definitions.

Proposition 2.7 If $\mathfrak{A}, \mathfrak{B} \in \mathscr{S}^{I}(U, E)$, then $\mathfrak{A} \widetilde{\subseteq}_{L} \mathfrak{B} \Rightarrow \mathfrak{A} \widetilde{\subseteq}_{J} \mathfrak{B}$.

Two IVF soft sets $\mathfrak{A}=(\widetilde{F}, A)$ and $\mathfrak{B}=(\widetilde{G}, B)$ are said to be identical, denoted by $\mathfrak{A} \equiv$ $\mathfrak{B}$, if they have the same parameter sets as well as approximate functions. That is, $A=B$ and $\widetilde{F}(a)=\widetilde{G}(a)$ for all $a \in A$. As an immediate consequence of Proposition 2.7 and the definition of IVF soft identical relations, we get a result as follows.

Corollary 2.8 Suppose that $\mathfrak{A}=(\widetilde{F}, A)$ and $\mathfrak{B}=(\widetilde{G}, B)$ are two IVF soft sets over $U$. Then we have

$$
\mathfrak{A} \equiv \mathfrak{B} \quad \Rightarrow \quad \mathfrak{A}={ }_{L} \mathfrak{B} \quad \Rightarrow \quad \mathfrak{A}={ }_{J} \mathfrak{B} .
$$

It is worth noting that all the reverse implications in Proposition 2.7 and Corollary $2.8 \mathrm{do}$ not hold in general. For more details, one can refer to the discussion regarding Molodtsov's soft sets in $[28,29]$. 
The above-mentioned concepts are useful in characterizing some fundamental algebraic properties of soft product operations $\wedge$ and $\vee$. To show this, we consider the following result.

Theorem 2.9 [30] (Generalized commutative laws of IVF soft sets) Let $(\widetilde{F}, A)$ and $(\widetilde{G}, B)$ be two IVF soft sets over $U$. Then we have

(1) $(\widetilde{F}, A) \wedge(\widetilde{G}, B)={ }_{L}(\widetilde{G}, B) \wedge(\widetilde{F}, A)$;

(2) $(\widetilde{F}, A) \vee(\widetilde{G}, B)=_{L}(\widetilde{G}, B) \vee(\widetilde{F}, A)$.

In view of results, we can see that the commutative laws do not hold in the conventional sense, which are characterized by IVF soft identical relation. Another important fact concerns algebraic properties regarding distributivity of soft product operations.

Theorem 2.10 [30] Let $(\widetilde{F}, A),(\widetilde{G}, B)$, and $(\widetilde{H}, C)$ be IVF soft sets over $U$. Then we have

(1) $((\widetilde{F}, A) \vee(\widetilde{G}, B)) \wedge(\widetilde{H}, C) \widetilde{\widetilde{C}}_{L}((\widetilde{F}, A) \wedge(\widetilde{H}, C)) \vee((\widetilde{G}, B) \wedge(\widetilde{H}, C))$;

(2) $((\widetilde{F}, A) \wedge(\widetilde{G}, B)) \vee(\widetilde{H}, C) \widetilde{\subseteq}_{L}((\widetilde{F}, A) \vee(\widetilde{H}, C)) \wedge((\widetilde{G}, B) \vee(\widetilde{H}, C))$.

In a similar fashion, Liu et al. [28] proposed the following distributive inequalities of IVF soft sets.

Theorem 2.11 Let $(\widetilde{F}, A),(\widetilde{G}, B)$, and $(\widetilde{H}, C)$ be IVF soft sets over $U$. Then we have

(1) $(\widetilde{F}, A) \wedge((\widetilde{G}, B) \vee(H, C)) \widetilde{\subseteq}_{L}((\widetilde{F}, A) \wedge(\widetilde{G}, B)) \vee((\widetilde{F}, A) \wedge(\widetilde{H}, C))$;

(2) $(\widetilde{F}, A) \vee((\widetilde{G}, B) \wedge(\widetilde{H}, C)) \widetilde{\subseteq}_{L}((\widetilde{F}, A) \vee(\widetilde{G}, B)) \wedge((\widetilde{F}, A) \vee(\widetilde{H}, C))$.

Remark 2.12 It is interesting to point out that the soft product operations of IVF soft sets possess some non-classical algebraic properties, as shown by the above results. Comparing with interval-valued fuzzy sets, we can find that $(\mathscr{I}(U), \cap, \cup)$ forms a distributive lattice, while only distributive inequalities (described by the IVF soft $L$-inclusion $\widetilde{\subseteq}_{L}$ ) hold for IVF soft sets. Thus neither the left nor the right distributive laws hold even in the weakest sense of IVF soft $J$-equal relations.

\section{Some basic properties of IVF soft inclusions}

Here we propose several basic inequalities of IVF soft sets characterized by IVF soft inclusions, which are useful in subsequent discussions.

Proposition 3.1 [30] Let $(\widetilde{F}, A)$ and $(\widetilde{G}, B)$ be IVF soft sets over $U$. Then we have

(1) $(\widetilde{F}, A) \widetilde{\widetilde{\subseteq}}_{J}(\widetilde{F}, A) \vee(\widetilde{G}, B)$;

(2) $(\widetilde{G}, B) \widetilde{\widetilde{\subseteq}}_{J}(\widetilde{F}, A) \vee(\widetilde{G}, B)$;

(3) $(\widetilde{F}, A) \wedge(\widetilde{G}, B) \widetilde{\subseteq}_{J}(\widetilde{F}, A)$;

(4) $(\widetilde{F}, A) \wedge(\widetilde{G}, B) \widetilde{\subseteq}_{J}(\widetilde{G}, B)$.

Proposition 3.2 [30] The IVF soft L-inclusion $\widetilde{\subseteq}_{L}$ is a preorder on $\mathscr{S}^{I}(U, E)$.

Proposition $3.3[30]$ The IVF soft J-inclusion $\tilde{\subseteq}_{J}$ is a preorder on $\mathscr{S}^{I}(U, E)$.

By generic properties of preorders, we can deduce the following two results.

Corollary 3.4 The IVF soft $L$-equal relation $=_{L}$ is an equivalence relation on $\mathscr{S}^{I}(U, E)$. 
Corollary 3.5 The IVF soft J-equal relation ${ }_{J}{ }_{J}$ is an equivalence relation on $\mathscr{S}^{I}(U, E)$.

Proposition 3.6 Let $(\widetilde{F}, A),(\widetilde{G}, B)$, and $(\widetilde{H}, C)$ be IVF soft sets over $U$. If $(\widetilde{F}, A) \widetilde{\subseteq}_{L}(\widetilde{G}, B)$, then we have

(1) $(\widetilde{H}, C) \vee(\widetilde{F}, A) \widetilde{\subseteq}_{L}(\widetilde{H}, C) \vee(\widetilde{G}, B)$;

(2) $(\widetilde{H}, C) \vee(\widetilde{F}, A) \widetilde{\subseteq}_{L}(\widetilde{G}, B) \vee(\widetilde{H}, C)$;

(3) $(\widetilde{F}, A) \vee(\widetilde{H}, C) \widetilde{\subseteq}_{L}(\widetilde{G}, B) \vee(\widetilde{H}, C)$;

(4) $(\widetilde{F}, A) \vee(\widetilde{H}, C) \widetilde{\subseteq}_{L}(\widetilde{H}, C) \vee(\widetilde{G}, B)$.

Proof To prove the first assertion, let $(\widetilde{H}, C) \vee(\widetilde{F}, A)=(\widetilde{L}, C \times A)$ and $(\widetilde{H}, C) \vee(\widetilde{G}, B)=$ $\left(\widetilde{R}_{1}, C \times B\right)$. By hypothesis, we have $(\widetilde{F}, A) \widetilde{\subseteq}_{L}(\widetilde{G}, B)$ and so for every $a \in A$ there exists $b \in B$ such that $\widetilde{F}(a)=\widetilde{G}(b)$. For any $(c, a) \in C \times A$, we deduce that

$$
\widetilde{L}(c, a)=\widetilde{H}(c) \cup \widetilde{F}(a)=\widetilde{H}(c) \cup \widetilde{G}(b)=\widetilde{R}_{1}(c, b),
$$

for some $(c, b) \in B \times C$. Hence $(\widetilde{H}, C) \vee(\widetilde{F}, A) \widetilde{\subseteq}_{L}(\widetilde{H}, C) \vee(\widetilde{G}, B)$.

Next, we show the second assertion. Let $(\widetilde{G}, B) \vee(\widetilde{H}, C)=\left(\widetilde{R}_{2}, B \times C\right)$. By the generalized commutative laws of IVF soft sets in Theorem 2.9, we have

$$
\left(\widetilde{R}_{2}, B \times C\right) \equiv(\widetilde{G}, B) \vee(\widetilde{H}, C)=_{L}(\widetilde{H}, C) \vee(\widetilde{G}, B) \equiv\left(\widetilde{R}_{1}, C \times B\right)
$$

Note also that we have verified that $(\widetilde{L}, C \times A) \widetilde{\subseteq}_{L}\left(\widetilde{R}_{1}, C \times B\right)$ in the first assertion. It follows that

$$
(\widetilde{L}, C \times A) \widetilde{\subseteq}_{L}\left(\widetilde{R}_{1}, C \times B\right) \widetilde{\subseteq}_{L}\left(\widetilde{R}_{2}, B \times C\right),
$$

by transitivity of the preorder $\widetilde{\subseteq}_{L}$ on $\mathscr{S}^{I}(U, E)$.

The proofs of the other two assertions can be obtained in a similar fashion and thus omitted.

Moreover, one can easily verify a result as follows.

Proposition 3.7 Let $\left(\widetilde{F}_{i}, A_{i}\right),\left(\widetilde{G}_{i}, B_{i}\right)$ be IVF soft sets over $U$ and $i=1$, 2 . If $\left(\widetilde{F}_{1}, A_{1}\right) \widetilde{\subseteq}_{L}\left(\widetilde{G}_{1}, B_{1}\right)$ and $\left(\widetilde{F}_{2}, A_{2}\right) \widetilde{\subseteq}_{L}\left(\widetilde{G}_{2}, B_{2}\right)$, then we have

$$
\left(\widetilde{F}_{1}, A_{1}\right) \vee\left(\widetilde{F}_{2}, A_{2}\right) \widetilde{\subseteq}_{L}\left(\widetilde{G}_{1}, B_{1}\right) \vee\left(\widetilde{G}_{2}, B_{2}\right)
$$

Considering $\wedge$-product of IVF soft sets, one can verify the following results which are analogous to Proposition 3.6 and 3.7, respectively.

Proposition 3.8 Let $(\widetilde{F}, A),(\widetilde{G}, B)$, and $(\widetilde{H}, C)$ be IVF soft sets over $U$. If $(\widetilde{F}, A) \widetilde{\subseteq}_{L}(\widetilde{G}, B)$, then we have

(1) $(\widetilde{H}, C) \wedge(\widetilde{F}, A) \widetilde{\subseteq}_{L}(\widetilde{H}, C) \wedge(\widetilde{G}, B)$;

(2) $(\widetilde{H}, C) \wedge(\widetilde{F}, A) \widetilde{\subseteq}_{L}(\widetilde{G}, B) \wedge(\widetilde{H}, C)$;

(3) $(\widetilde{F}, A) \wedge(\widetilde{H}, C) \widetilde{\subseteq}_{L}(\widetilde{G}, B) \wedge(\widetilde{H}, C)$;

(4) $(\widetilde{F}, A) \wedge(\widetilde{H}, C) \widetilde{\subseteq}_{L}(\widetilde{H}, C) \wedge(\widetilde{G}, B)$. 
Proposition 3.9 Let $\left(\widetilde{F}_{i}, A_{i}\right),\left(\widetilde{G}_{i}, B_{i}\right)$ be IVF soft sets over $U$ and $i=1,2$. If $\left(\widetilde{F}_{1}, A_{1}\right) \widetilde{\subseteq}_{L}\left(\widetilde{G}_{1}, B_{1}\right)$ and $\left(\widetilde{F}_{2}, A_{2}\right) \widetilde{\subseteq}_{L}\left(\widetilde{G}_{2}, B_{2}\right)$, then we have

$$
\left(\widetilde{F}_{1}, A_{1}\right) \wedge\left(\widetilde{F}_{2}, A_{2}\right) \widetilde{\subseteq}_{L}\left(\widetilde{G}_{1}, B_{1}\right) \wedge\left(\widetilde{G}_{2}, B_{2}\right)
$$

Regarding Jun's inclusion $\widetilde{\subseteq}_{J}$ of IVF soft sets, we obtained the following similar results in $[30]$.

Proposition 3.10 Let $(\widetilde{F}, A),(\widetilde{G}, B)$ and $(\widetilde{H}, C)$ be IVF soft sets over $U$. If $(\widetilde{F}, A) \widetilde{\subseteq}_{J}(\widetilde{G}, B)$, then we have

(1) $(\widetilde{F}, A) \wedge(\widetilde{H}, C) \widetilde{\subseteq}_{J}(\widetilde{G}, B) \wedge(\widetilde{H}, C)$;

(2) $(\widetilde{F}, A) \wedge(\widetilde{H}, C) \widetilde{\widetilde{C}}_{J}(\widetilde{H}, C) \wedge(\widetilde{G}, B)$;

(3) $(\widetilde{H}, C) \wedge(\widetilde{F}, A) \widetilde{\subseteq}_{J}(\widetilde{H}, C) \wedge(\widetilde{G}, B)$;

(4) $(\widetilde{H}, C) \wedge(\widetilde{F}, A) \widetilde{\subseteq}_{J}(\widetilde{G}, B) \wedge(\widetilde{H}, C)$;

(5) $(\widetilde{F}, A) \vee(\widetilde{H}, C) \widetilde{\subseteq}_{J}(\widetilde{G}, B) \vee(\widetilde{H}, C)$;

(6) $(\widetilde{F}, A) \vee(\widetilde{H}, C) \widetilde{\widetilde{\subseteq}}_{J}(\tilde{H}, C) \vee(\widetilde{G}, B)$

(7) $(\widetilde{H}, C) \vee(\widetilde{F}, A) \widetilde{\subseteq}_{J}(\widetilde{H}, C) \vee(\widetilde{G}, B)$;

(8) $(\tilde{H}, C) \vee(\widetilde{F}, A) \widetilde{\subseteq}_{J}(\widetilde{G}, B) \vee(\widetilde{H}, C)$.

Proposition 3.11 Let $\left(\widetilde{F}_{i}, A_{i}\right),\left(\widetilde{G}_{i}, B_{i}\right)$ be IVF soft sets over $U$ and $i=1,2$. If $\left(\widetilde{F}_{1}, A_{1}\right) \widetilde{\subseteq}_{J}\left(\widetilde{G}_{1}\right.$, $\left.B_{1}\right)$ and $\left(\widetilde{F}_{2}, A_{2}\right) \widetilde{\subseteq}_{J}\left(\widetilde{G}_{2}, B_{2}\right)$, then we have

$$
\left(\widetilde{F}_{1}, A_{1}\right) \wedge\left(\widetilde{F}_{2}, A_{2}\right) \widetilde{\subseteq}_{J}\left(\widetilde{G}_{1}, B_{1}\right) \wedge\left(\widetilde{G}_{2}, B_{2}\right),
$$

and

$$
\left(\widetilde{F}_{1}, A_{1}\right) \vee\left(\widetilde{F}_{2}, A_{2}\right) \widetilde{\subseteq}_{J}\left(\widetilde{G}_{1}, B_{1}\right) \vee\left(\widetilde{G}_{2}, B_{2}\right)
$$

\section{Upward directed IVF soft sets and idempotency}

Now, we investigate algebraic properties of soft product operations of IVF soft sets by considering idempotency. First, we recall some important results proposed by Liu et al. [30].

Proposition 4.1 [30] Let $(\widetilde{F}, A) \in \mathscr{S}^{I}(U, E)$. Then $(\widetilde{F}, A) \widetilde{\subseteq}_{L}(\widetilde{F}, A) \vee(\widetilde{F}, A)$.

Proposition $4.2[30] \operatorname{Let}(\widetilde{F}, A) \in \mathscr{S}^{I}(U, E)$. Then $(\widetilde{F}, A) \widetilde{\subseteq}_{L}(\widetilde{F}, A) \wedge(\widetilde{F}, A)$.

Theorem 4.3 [30] Let $(\widetilde{F}, A) \in \mathscr{S}^{I}(U, E)$. Then $(\widetilde{F}, A)=_{J}(\widetilde{F}, A) \wedge(\widetilde{F}, A)$.

The last result indicates that the $\wedge$-product operation of IVF soft sets is idempotent with respect to IVF soft $J$-equal relations, and is referred to as the weak idempotent law of IVF soft sets. Nevertheless, the parallel result regarding $\vee$-product operations of IVF soft sets does not hold.

We know that $\cap$ and $\cup$ are both idempotent since $(\mathscr{I}(U), \cap, \cup)$ is a lattice in the theory of interval-valued fuzzy sets. The two operations $\cap$ and $\cup$ are dual to each other, which always satisfy similar or parallel algebraic properties. According to Definition 2.3 and Definition 2.4, the operations $\wedge$ and $\vee$ of IVF soft sets are defined in terms of the intersection 
$\cap$ and union $\cup$ of interval-valued fuzzy sets, respectively. Nevertheless, it is interesting to see that the operations $\wedge$ and $\vee$ of IVF soft sets do not always have similar algebraic properties. In fact, as shown by some illustrative examples in [30] we have

$$
(\widetilde{F}, A)=_{J}(\widetilde{F}, A) \wedge(\widetilde{F}, A)
$$

but

$$
(\widetilde{F}, A) \neq_{J}(\widetilde{F}, A) \vee(\widetilde{F}, A) .
$$

Note also that

$$
(\widetilde{F}, A) \neq_{L}(\widetilde{F}, A) \wedge(\widetilde{F}, A)
$$

which shows that the $\wedge$-product operation of IVF soft sets is idempotent with respect to the IVF soft $J$-equal relation $=_{J}$, but not in the stronger sense of $=_{L}$. Thus considering the IVF soft $L$-inclusion $\widetilde{\subseteq}_{L}$, we only have some idempotent inequalities as shown in Proposition 4.1 and Proposition 4.2. In view of these results, we conclude that IVF soft sets possess some non-classical algebraic properties, compared with interval-valued fuzzy sets. The interested readers could refer to $[28,30]$ for more details.

Recall that a nonempty set $A$ together with a preorder $\leq$ is called an upward directed set if every pair of elements in $A$ has an upper bound. That is, for every $a, b \in A$, there exists $c \in A$ such that $a \leq c$ and $b \leq c$.

Definition 4.4 Let $(\widetilde{F}, A)$ be an IVF soft set over $U$ with $A \neq \emptyset$. Then $(\widetilde{F}, A)$ is said to be upward directed if for every $a_{1}, a_{2} \in A$, there exists $a_{3} \in A$ such that

$$
\widetilde{F}\left(a_{1}\right) \cup \widetilde{F}\left(a_{2}\right) \subseteq \widetilde{F}\left(a_{3}\right) .
$$

Example 4.5 Let $U=\{a, b, c, d, e\}$ be the universe and the parameter space $E=\left\{e_{1}, e_{2}, e_{3}\right.$, $\left.e_{4}, e_{5}\right\}$. Assume that the parameter set $A=\left\{e_{1}, e_{2}, e_{4}\right\}$ and $\mathfrak{A}=(\widetilde{F}, A)$ is an IVF soft set over $U$ with tabular representation given by Table 1 . By definition, one can verify that $\mathfrak{A}=(\widetilde{F}, A)$ is an upward directed IVF soft set over $U$.

The following statements justify the term upward directed IVF soft sets and illustrate some intuitive ideas for introducing such a notion in the theory of IVF soft sets.

Proposition 4.6 Let $\mathfrak{S}=(\widetilde{F}, A) \in \mathscr{S}^{I}(U, E)$ and $C_{\mathfrak{S}}=\{\widetilde{F}(a) \mid a \in A\}$. Then $\mathfrak{S}$ is an upward directed IVF soft set over $U$ if and only if $\left(C_{\mathfrak{S}}, \subseteq\right)$ is an upward directed set.

Table 1 Tabular representation of the IVF soft set $\mathfrak{A}=(\tilde{F}, A)$

\begin{tabular}{llll}
\hline $\boldsymbol{U}$ & $\boldsymbol{e}_{\mathbf{1}}$ & $\boldsymbol{e}_{\mathbf{2}}$ & $\boldsymbol{e}_{\mathbf{4}}$ \\
\hline$a$ & {$[0.4,0.5]$} & {$[0.2,0.6]$} & {$[0.5,0.8]$} \\
$b$ & {$[0.2,0.6]$} & {$[0.3,0.5]$} & {$[0.3,0.7]$} \\
$c$ & {$[0.3,0.4]$} & {$[0.5,0.6]$} & {$[0.6,0.7]$} \\
$d$ & {$[0.5,0.7]$} & {$[0.6,0.9]$} & {$[0.7,0.9]$} \\
$e$ & {$[0.2,0.6]$} & {$[0.1,0.5]$} & {$[0.4,0.8]$} \\
\hline
\end{tabular}


Proof First, suppose that $\mathfrak{S}=(\widetilde{F}, A)$ is an upward directed IVF soft set over $U$. Then by the definition of upward directed IVF soft sets, we have $A \neq \emptyset$ and so $C_{\mathfrak{S}}=\{\widetilde{F}(a) \mid a \in A\}$ is a nonempty subset of $\mathscr{I}(U)$. Moreover, for every $a_{1}, a_{2} \in A$, there exists $a_{3} \in A$ such that $\widetilde{F}\left(a_{1}\right) \cup \widetilde{F}\left(a_{2}\right) \subseteq \widetilde{F}\left(a_{3}\right)$. It follows that $\widetilde{F}\left(a_{1}\right) \subseteq \widetilde{F}\left(a_{3}\right)$ and $\widetilde{F}\left(a_{2}\right) \subseteq \widetilde{F}\left(a_{3}\right)$. This shows that $C_{\mathfrak{S}}$ is an upward directed set with respect to the partial order $\subseteq$.

Conversely, let $\left(C_{\mathfrak{S}}, \subseteq\right)$ be an upward directed set. Then $A \neq \emptyset$ since $C_{\mathfrak{S}}$ is nonempty. For every $a_{1}, a_{2} \in A$, by the definition of upward directed sets, the pair of elements $\widetilde{F}\left(a_{1}\right)$ and $\widetilde{F}\left(a_{2}\right)$ has an upper bound in $C_{\mathfrak{S}}$. Hence there exists $a_{3} \in A$ such that $\widetilde{F}\left(a_{1}\right) \subseteq \widetilde{F}\left(a_{3}\right)$ and $\widetilde{F}\left(a_{2}\right) \subseteq \widetilde{F}\left(a_{3}\right)$. It follows that $\widetilde{F}\left(a_{1}\right) \cup \widetilde{F}\left(a_{2}\right) \subseteq \widetilde{F}\left(a_{3}\right)$. Therefore, $\mathfrak{S}=(\widetilde{F}, A)$ is an upward directed IVF soft set over $U$.

Proposition 4.7 Let $(\widetilde{F}, A) \in \mathscr{S}^{I}(U, E)$ and $A \neq \emptyset$. Then $(\widetilde{F}, A)$ is an upward directed IVF soft set over $U$ if and only if $(\widetilde{F}, A)={ }_{J}(\widetilde{F}, A) \vee(\widetilde{F}, A)$.

Proof Let us denote $(\widetilde{F}, A) \vee(\widetilde{F}, A)$ by $(\widetilde{R}, A \times A)$. First, assume that $(\widetilde{F}, A)$ is an upward directed IVF soft set over $U$. For every $\left(a_{1}, a_{2}\right) \in A \times A$, there exists $a_{3} \in A$ such that

$$
\widetilde{R}\left(a_{1}, a_{2}\right)=\widetilde{F}\left(a_{1}\right) \cup \widetilde{F}\left(a_{2}\right) \subseteq \widetilde{F}\left(a_{3}\right),
$$

since $(\widetilde{F}, A)$ is upward directed. This shows that

$$
(\widetilde{F}, A) \vee(\widetilde{F}, A) \widetilde{\subseteq}_{J}(\widetilde{F}, A) .
$$

But by Proposition 4.3 , we also have $(\widetilde{F}, A) \widetilde{\subseteq}_{L}(\widetilde{F}, A) \vee(\widetilde{F}, A)$, and so

$$
(\widetilde{F}, A) \widetilde{\subseteq}_{J}(\widetilde{F}, A) \vee(\widetilde{F}, A) .
$$

Hence we deduce that $(\widetilde{F}, A)={ }_{J}(\widetilde{F}, A) \vee(\widetilde{F}, A)$.

Conversely, let $(\widetilde{F}, A)=_{J}(\widetilde{F}, A) \vee(\widetilde{F}, A)$. Then in particular, we have

$$
(\widetilde{R}, A \times A)=(\widetilde{F}, A) \vee(\widetilde{F}, A) \widetilde{\subseteq}_{J}(\widetilde{F}, A) .
$$

Now for every $a_{1}, a_{2} \in A$, there exists $a_{3} \in A$ such that

$$
\widetilde{F}\left(a_{1}\right) \cup \widetilde{F}\left(a_{2}\right)=\widetilde{R}\left(a_{1}, a_{2}\right) \subseteq \widetilde{F}\left(a_{3}\right) .
$$

Hence by definition, $(\widetilde{F}, A)$ is an upward directed IVF soft set over $U$.

Corollary 4.8 Let $\mathfrak{S}=(\widetilde{F}, A) \in \mathscr{S}^{I}(U, E)$ and $A \neq \emptyset$. Then the following are equivalent:

(1) $\mathfrak{S}$ is an upward directed IVF soft set over $U$.

(2) $C_{\mathfrak{S}}=\{\widetilde{F}(a) \mid a \in A\}$ is an upward directed set with respect to $\subseteq$.

(3) $(\widetilde{F}, A) \vee(\widetilde{F}, A)==_{J}(\widetilde{F}, A)$.

(4) $(\widetilde{F}, A) \vee(\widetilde{F}, A) \widetilde{\subseteq}_{J}(\widetilde{F}, A)$.

Proof According to Proposition 4.6 and Proposition 4.7, we have (1) $\Leftrightarrow(2)$ and (1) $\Leftrightarrow(3)$, respectively. Thus we only need to show that $(1) \Leftrightarrow(4)$. In fact, let us denote $(\widetilde{F}, A) \vee(\widetilde{F}, A)$ 
by $(\widetilde{G}, A \times A)$. Then by Definition 4.4, $(\widetilde{F}, A)$ is an upward directed IVF soft set over $U$ if and only if for every $a_{1}, a_{2} \in A$, there exists $a_{3} \in A$ such that

$$
\widetilde{G}\left(a_{1}, a_{2}\right)=\widetilde{F}\left(a_{1}\right) \cup \widetilde{F}\left(a_{2}\right) \subseteq \widetilde{F}\left(a_{3}\right) .
$$

Clearly, this is equivalent to $(\widetilde{G}, A \times A) \widetilde{\subseteq}_{J}(\widetilde{F}, A)$, completing our proof.

\section{Modular inequalities of IVF soft sets}

Let $(L, \wedge, \vee, \leq)$ be a lattice and $a, b, c \in L$. Then one can verify that

$$
a \leq c \quad \Rightarrow \quad a \vee(b \wedge c) \leq(a \vee b) \wedge c .
$$

In particular, for every interval-valued fuzzy sets $\mu, v, \lambda$, one can deduce that

$$
\mu \subseteq \lambda \quad \Rightarrow \quad \mu \cup(v \cap \lambda) \subseteq(\mu \cup v) \cap \lambda
$$

Actually, the modular law

$$
\mu \subseteq \lambda \Rightarrow \mu \cup(\nu \cap \lambda)=(\mu \cup v) \cap \lambda
$$

holds for all $\mu, \nu, \lambda \in \mathscr{I}(U)$ since $(\mathscr{I}(U), \cap, \cup)$ forms a distributive lattice. Considering IVF soft sets and soft product operations, we encounter a situation in contrast to the above. First, we have following types of modular inequalities of IVF soft sets.

Theorem 5.1 Let $(\widetilde{F}, A),(\widetilde{G}, B)$, and $(\widetilde{H}, C)$ be IVF soft sets over $U$. Then we have

$$
((\widetilde{F}, A) \vee(\widetilde{G}, B)) \wedge(\widetilde{H}, C) \widetilde{\subseteq}_{J}(\widetilde{F}, A) \vee((\widetilde{G}, B) \wedge(\widetilde{H}, C))
$$

Proof By the distributive inequalities in Theorem 2.10, we have

$$
((\widetilde{F}, A) \vee(\widetilde{G}, B)) \wedge(\widetilde{H}, C) \widetilde{\subseteq}_{L}((\widetilde{F}, A) \wedge(\widetilde{H}, C)) \vee((\widetilde{G}, B) \wedge(\widetilde{H}, C)),
$$

and it implies that

$$
((\widetilde{F}, A) \vee(\widetilde{G}, B)) \wedge(\widetilde{H}, C) \widetilde{\subseteq}_{J}((\widetilde{F}, A) \wedge(\widetilde{H}, C)) \vee((\widetilde{G}, B) \wedge(\widetilde{H}, C)) .
$$

Note also that

$$
(\widetilde{F}, A) \wedge(\widetilde{H}, C) \widetilde{\subseteq}_{J}(\widetilde{F}, A)
$$

by Proposition 3.1. Thus from Proposition 3.10, it follows that

$$
((\widetilde{F}, A) \wedge(\widetilde{H}, C)) \vee((\widetilde{G}, B) \wedge(\widetilde{H}, C)) \widetilde{\subseteq}_{J}(\widetilde{F}, A) \vee((\widetilde{G}, B) \wedge(\widetilde{H}, C)) .
$$

Since $\widetilde{\subseteq}_{J}$ is transitive on $\mathscr{S}^{I}(U, E)$, we deduce that

$$
((\widetilde{F}, A) \vee(\widetilde{G}, B)) \wedge(\widetilde{H}, C) \widetilde{\subseteq}_{J}(\widetilde{F}, A) \vee((\widetilde{G}, B) \wedge(\widetilde{H}, C)),
$$

which completes the proof. 
It is interesting to see that the reverse soft $J$-inclusion does not hold in general. Actually we will illustrate this fact with an example in the following. Using the generalized commutative laws of IVF soft sets in Theorem 2.9, we have the following consequences of Theorem 5.1.

Corollary 5.2 Let $(\widetilde{F}, A),(\widetilde{G}, B)$ and $(\widetilde{H}, C)$ be IVF soft sets over $U$. Then we have

(1) $((\widetilde{G}, B) \vee(\widetilde{F}, A)) \wedge(\widetilde{H}, C) \widetilde{\subseteq}_{J}(\widetilde{F}, A) \vee((\widetilde{G}, B) \wedge(\widetilde{H}, C))$;

(2) $((\widetilde{F}, A) \vee(\widetilde{G}, B)) \wedge(\widetilde{H}, C) \widetilde{\subseteq}_{J}(\widetilde{F}, A) \vee((\widetilde{H}, C) \wedge(\widetilde{G}, B))$;

(3) $((\widetilde{G}, B) \vee(\widetilde{F}, A)) \wedge(\widetilde{H}, C) \widetilde{\subseteq}_{J}(\widetilde{F}, A) \vee((\widetilde{H}, C) \wedge(\widetilde{G}, B))$.

Proof We only prove the first soft $J$-inclusion; the proofs of other soft $J$-inclusions can be obtained using similar techniques. Note first that

$$
(\widetilde{G}, B) \vee(\widetilde{F}, A)={ }_{L}(\widetilde{F}, A) \vee(\widetilde{G}, B),
$$

by the generalized commutative laws of IVF soft sets. It follows that

$$
(\widetilde{G}, B) \vee(\widetilde{F}, A) \widetilde{\subseteq}_{J}(\widetilde{F}, A) \vee(\widetilde{G}, B)
$$

Thus we deduce that

$$
((\widetilde{G}, B) \vee(\widetilde{F}, A)) \wedge(\widetilde{H}, C) \widetilde{\subseteq}_{J}((\widetilde{F}, A) \vee(\widetilde{G}, B)) \wedge(\widetilde{H}, C)
$$

according to Proposition 3.10. But by Theorem 5.1, we also have

$$
((\widetilde{F}, A) \vee(\widetilde{G}, B)) \wedge(\widetilde{H}, C) \widetilde{\subseteq}_{J}(\widetilde{F}, A) \vee((\widetilde{G}, B) \wedge(\widetilde{H}, C))
$$

Hence we conclude that

$$
((\widetilde{G}, B) \vee(\widetilde{F}, A)) \wedge(\widetilde{H}, C) \widetilde{\subseteq}_{J}(\widetilde{F}, A) \vee((\widetilde{G}, B) \wedge(\widetilde{H}, C))
$$

since $\widetilde{\subseteq}_{J}$ is transitive on $\mathscr{S}^{I}(U, E)$.

In a similar fashion, one can verify the following statements.

Corollary 5.3 Let $(\widetilde{F}, A),(\widetilde{G}, B)$ and $(\widetilde{H}, C)$ be IVF soft sets over $U$. Then we have

(1) $(\widetilde{H}, C) \wedge((\widetilde{G}, B) \vee(\widetilde{F}, A)) \widetilde{\subseteq}_{J}(\widetilde{F}, A) \vee((\widetilde{G}, B) \wedge(\widetilde{H}, C))$;

(2) $(\widetilde{H}, C) \wedge((\widetilde{F}, A) \vee(\widetilde{G}, B)) \widetilde{\subseteq}_{J}(\widetilde{F}, A) \vee((\widetilde{H}, C) \wedge(\widetilde{G}, B))$;

(3) $(\widetilde{H}, C) \wedge((\widetilde{G}, B) \vee(\widetilde{F}, A)) \underline{\widetilde{\subseteq}}_{J}(\widetilde{F}, A) \vee((\widetilde{H}, C) \wedge(\widetilde{G}, B))$;

(4) $(\tilde{H}, C) \wedge((\widetilde{F}, A) \vee(\widetilde{G}, B)) \widetilde{\subseteq}_{J}(\widetilde{F}, A) \vee((\widetilde{G}, B) \wedge(\tilde{H}, C))$.

Corollary 5.4 Let $(\widetilde{F}, A),(\widetilde{G}, B)$, and $(\widetilde{H}, C)$ be IVF soft sets over $U$. Then we have

(1) $(\widetilde{H}, C) \wedge((\widetilde{G}, B) \vee(\widetilde{F}, A)) \widetilde{\subseteq}_{J}((\widetilde{G}, B) \wedge(\widetilde{H}, C)) \vee(\widetilde{F}, A)$;

(2) $(\widetilde{H}, C) \wedge((\widetilde{F}, A) \vee(\widetilde{G}, B)) \widetilde{\subseteq}_{J}((\widetilde{H}, C) \wedge(\widetilde{G}, B)) \vee(\widetilde{F}, A)$;

(3) $(\widetilde{H}, C) \wedge((\widetilde{G}, B) \vee(\widetilde{F}, A)) \widetilde{\subseteq}_{J}((\widetilde{H}, C) \wedge(\widetilde{G}, B)) \vee(\widetilde{F}, A)$;

(4) $(\widetilde{H}, C) \wedge((\widetilde{F}, A) \vee(\widetilde{G}, B)) \widetilde{\subseteq}_{J}((\widetilde{G}, B) \wedge(\widetilde{H}, C)) \vee(\widetilde{F}, A)$. 
Corollary 5.5 Let $(\widetilde{F}, A),(\widetilde{G}, B)$, and $(\widetilde{H}, C)$ be IVF soft sets over $U$. Then we have

(1) $((\widetilde{G}, B) \vee(\widetilde{F}, A)) \wedge(\widetilde{H}, C) \widetilde{\subseteq}_{J}((\widetilde{G}, B) \wedge(\widetilde{H}, C)) \vee(\widetilde{F}, A)$;

(2) $((\widetilde{F}, A) \vee(\widetilde{G}, B)) \wedge(\widetilde{H}, C) \widetilde{\subseteq}_{J}((\widetilde{H}, C) \wedge(\widetilde{G}, B)) \vee(\widetilde{F}, A)$;

(3) $((\widetilde{G}, B) \vee(\widetilde{F}, A)) \wedge(\widetilde{H}, C) \widetilde{\widetilde{S}}_{J}((\widetilde{H}, C) \wedge(\widetilde{G}, B)) \vee(\widetilde{F}, A)$;

(4) $((\widetilde{F}, A) \vee(\widetilde{G}, B)) \wedge(\widetilde{H}, C) \widetilde{\subseteq}_{J}((\widetilde{G}, B) \wedge(\widetilde{H}, C)) \vee(\widetilde{F}, A)$.

Theorem 5.6 Let $(\widetilde{F}, A),(\widetilde{G}, B)$, and $(\widetilde{H}, C)$ be IVF soft sets over $U$.If $(\widetilde{F}, A) \widetilde{\subseteq}_{J}(\widetilde{H}, C)$, then we have

$$
(\widetilde{F}, A) \vee((\widetilde{G}, B) \wedge(\widetilde{H}, C)) \widetilde{\subseteq}_{J}((\widetilde{F}, A) \vee(\widetilde{G}, B)) \wedge((\widetilde{H}, C) \vee(\widetilde{H}, C))
$$

Proof By the distributive inequalities in Theorem 2.11, we have

$$
(\widetilde{F}, A) \vee((\widetilde{G}, B) \wedge(\widetilde{H}, C)) \widetilde{\subseteq}_{L}((\widetilde{F}, A) \vee(\widetilde{G}, B)) \wedge((\widetilde{F}, A) \vee(\widetilde{H}, C))
$$

and so

$$
(\widetilde{F}, A) \vee((\widetilde{G}, B) \wedge(\widetilde{H}, C)) \widetilde{\subseteq}_{J}((\widetilde{F}, A) \vee(\widetilde{G}, B)) \wedge((\widetilde{F}, A) \vee(\widetilde{H}, C))
$$

Since $(\widetilde{F}, A) \widetilde{\subseteq}_{J}(\widetilde{H}, C)$ by the hypothesis, we deduce that

$$
(\widetilde{F}, A) \vee(\widetilde{H}, C) \widetilde{\subseteq}_{J}(\widetilde{H}, C) \vee(\widetilde{H}, C),
$$

by Proposition 3.10. Then it follows that

$$
((\widetilde{F}, A) \vee(\widetilde{G}, B)) \wedge((\widetilde{F}, A) \vee(\widetilde{H}, C)) \widetilde{\subseteq}_{J}((\widetilde{F}, A) \vee(\widetilde{G}, B)) \wedge((\widetilde{H}, C) \vee(\widetilde{H}, C))
$$

Consequently, we can obtain

$$
(\widetilde{F}, A) \vee((\widetilde{G}, B) \wedge(\widetilde{H}, C)) \widetilde{\subseteq}_{J}((\widetilde{F}, A) \vee(\widetilde{G}, B)) \wedge((\widetilde{H}, C) \vee(\widetilde{H}, C))
$$

by transitivity of the preorder $\widetilde{\subseteq}_{J}$ on $\mathscr{S}^{I}(U, E)$.

Example 5.7 Suppose that $U=\left\{h_{1}, h_{2}, h_{3}, h_{4}, h_{5}, h_{6}\right\}$ is the universe and $E=\left\{e_{1}, e_{2}, e_{3}\right.$, $\left.e_{4}, e_{5}\right\}$ is the parameter space. For the parameter sets $A=\left\{e_{1}, e_{2}\right\}, B=\left\{e_{3}\right\}$, and $C=\left\{e_{4}, e_{5}\right\}$, let $\mathfrak{S}=(\widetilde{F}, A), \mathfrak{T}=(\widetilde{G}, B)$, and $\mathfrak{K}=(\widetilde{H}, C)$ be three IVF soft sets over $U$, where

$$
\begin{aligned}
& \widetilde{F}\left(e_{1}\right)=\left\{\left(h_{1},[0.4,0.7]\right),\left(h_{2},[0.2,0.4]\right),\left(h_{3},[0.1,0.2]\right),\right.\left.\left(h_{4},[0.8,0.9]\right),\left(h_{5},[0.3,0.5]\right),\left(h_{6},[0.2,0.6]\right)\right\}, \\
& \widetilde{F}\left(e_{2}\right)=\left\{\left(h_{1},[0.2,0.6]\right),\left(h_{2},[0.1,0.6]\right),\left(h_{3},[0.5,0.7]\right),\right. \\
&\left.\left(h_{4},[0.6,0.9]\right),\left(h_{5},[0.6,0.7]\right),\left(h_{6},[0.4,0.5]\right)\right\}, \\
& \widetilde{G}\left(e_{3}\right)=\left\{\left(h_{1},[0.5,0.8]\right),\left(h_{2},[0.4,0.8]\right),\left(h_{3},[0.3,0.5]\right),\right. \\
&\left.\left(h_{4},[0.1,0.2]\right),\left(h_{5},[0.4,0.5]\right),\left(h_{6},[0.2,0.3]\right)\right\},
\end{aligned}
$$




$$
\begin{aligned}
\widetilde{H}\left(e_{4}\right)= & \left\{\left(h_{1},[0.8,0.9]\right),\left(h_{2},[0.2,0.5]\right),\left(h_{3},[0.2,0.4]\right),\right. \\
& \left.\left(h_{4},[0.8,0.9]\right),\left(h_{5},[0.3,0.5]\right),\left(h_{6},[0.3,0.6]\right)\right\}, \\
\widetilde{H}\left(e_{5}\right)= & \left\{\left(h_{1},[0.4,0.6]\right),\left(h_{2},[0.3,0.7]\right),\left(h_{3},[0.6,0.8]\right),\right. \\
& \left.\left(h_{4},[0.8,0.9]\right),\left(h_{5},[0.7,0.8]\right),\left(h_{6},[0.5,0.7]\right)\right\} .
\end{aligned}
$$

It is clear that $(\widetilde{F}, A) \widetilde{\subseteq}_{J}(\widetilde{H}, C)$ since $\widetilde{F}\left(e_{1}\right) \subseteq \widetilde{H}\left(e_{4}\right)$ and $\widetilde{F}\left(e_{2}\right) \subseteq \widetilde{H}\left(e_{5}\right)$. Let us write $(\widetilde{T}, B \times$ $C)$ for $(\widetilde{G}, B) \wedge(\widetilde{H}, C)$ where $\widetilde{T}(b, c)=\widetilde{G}(b) \cap \widetilde{H}(c)$ for all $(b, c) \in B \times C$. Then $B \times C=$ $\left\{\left(e_{3}, e_{4}\right),\left(e_{3}, e_{5}\right)\right\}$ and by calculation, one obtains

$$
\begin{aligned}
\widetilde{T}\left(e_{3}, e_{4}\right)= & \widetilde{G}\left(e_{3}\right) \cap \widetilde{H}\left(e_{4}\right)=\left\{\left(h_{1},[0.5,0.8]\right),\left(h_{2},[0.2,0.5),\left(h_{3},[0.2,0.4]\right),\right.\right. \\
& \left.\left(h_{4},[0.1,0.2]\right),\left(h_{5},[0.3,0.5]\right),\left(h_{6},[0.2,0.3]\right)\right\},
\end{aligned}
$$

and

$$
\begin{aligned}
& \widetilde{T}\left(e_{3}, e_{5}\right)=\widetilde{G}\left(e_{3}\right) \cap \widetilde{H}\left(e_{5}\right)=\left\{\left(h_{1},[0.4,0.6]\right),\left(h_{2},[0.3,0.7]\right),\left(h_{3},[0.3,0.5]\right),\right. \\
&\left.\left(h_{4},[0.1,0.2]\right),\left(h_{5},[0.4,0.5]\right),\left(h_{6},[0.2,0.3]\right)\right\} .
\end{aligned}
$$

Then let us write $(\widetilde{F}, A) \vee(\widetilde{T}, B \times C)$ as $\mathfrak{L}=(\widetilde{L}, A \times(B \times C))$, where

$$
\widetilde{L}(a,(b, c))=\widetilde{F}(a) \cup \widetilde{T}(b, c)=\widetilde{F}(a) \cup(\widetilde{G}(b) \cap \widetilde{H}(c))
$$

for all $(a,(b, c)) \in A \times(B \times C)$. It is easy to see that

$$
A \times(B \times C)=\left\{\left(e_{1},\left(e_{3}, e_{4}\right)\right),\left(e_{1},\left(e_{3}, e_{5}\right)\right),\left(e_{2},\left(e_{3}, e_{4}\right)\right),\left(e_{2},\left(e_{3}, e_{5}\right)\right)\right\}
$$

Proceeding with detailed calculations, one can obtain the IVF soft set $\mathfrak{L}$ with its tabular representation shown in Table 2 .

Next, we write $(\widetilde{M}, A \times B)$ for $(\widetilde{F}, A) \vee(\widetilde{G}, B)$ where $\widetilde{M}(a, b)=\widetilde{F}(a) \cup \widetilde{G}(b)$ for all $(a, b) \in$ $A \times B$. Let us denote $(\widetilde{H}, C) \vee(\widetilde{H}, C)$ by $\mathfrak{N}=(\widetilde{N}, C \times C)$, where $\widetilde{N}\left(c_{1}, c_{2}\right)=\widetilde{H}\left(c_{1}\right) \cup \widetilde{H}\left(c_{2}\right)$ for all $\left(c_{1}, c_{2}\right) \in C \times C$. It is easy to see that $A \times B=\left\{\left(e_{1}, e_{3}\right),\left(e_{2}, e_{3}\right)\right\}$ and $C \times C=$ $\left\{\left(e_{4}, e_{4}\right),\left(e_{4}, e_{5}\right),\left(e_{5}, e_{4}\right),\left(e_{5}, e_{5}\right)\right\}$. By calculation, we get

$$
\begin{aligned}
\widetilde{M}\left(e_{1}, e_{3}\right)= & \widetilde{F}\left(e_{1}\right) \cup \widetilde{G}\left(e_{3}\right)=\left\{\left(h_{1},[0.5,0.8]\right),\left(h_{2},[0.4,0.8),\left(h_{3},[0.3,0.5]\right),\right.\right. \\
& \left.\left(h_{4},[0.8,0.9]\right),\left(h_{5},[0.4,0.5]\right),\left(h_{6},[0.2,0.6]\right)\right\},
\end{aligned}
$$

and

$$
\begin{aligned}
\widetilde{M}\left(e_{2}, e_{3}\right)= & \widetilde{F}\left(e_{2}\right) \cup \widetilde{G}\left(e_{3}\right)=\left\{\left(h_{1},[0.5,0.8]\right),\left(h_{2},[0.4,0.8]\right),\left(h_{3},[0.5,0.7]\right),\right. \\
& \left.\left(h_{4},[0.6,0.9]\right),\left(h_{5},[0.6,0.7]\right),\left(h_{6},[0.4,0.5]\right)\right\} .
\end{aligned}
$$

Also we can obtain the IVF soft set $\mathfrak{N}=(\widetilde{N}, C \times C)$ with its tabular representation given by Table 3 . 
Table 2 Tabular representation of the IVF soft set $\mathfrak{L}=(\tilde{L}, A \times(B \times C))$

\begin{tabular}{lllllll}
\hline $\boldsymbol{U}$ & $\boldsymbol{h}_{\mathbf{1}}$ & $\boldsymbol{h}_{\mathbf{2}}$ & $\boldsymbol{h}_{\mathbf{3}}$ & $\boldsymbol{h}_{\mathbf{4}}$ & $\boldsymbol{h}_{\mathbf{5}}$ & $\boldsymbol{h}_{\mathbf{6}}$ \\
\hline$\left(e_{1},\left(e_{3}, e_{4}\right)\right)$ & {$[0.5,0.8]$} & {$[0.2,0.5]$} & {$[0.2,0.4]$} & {$[0.8,0.9]$} & {$[0.3,0.5]$} & {$[0.2,0.6]$} \\
$\left(e_{1},\left(e_{3}, e_{5}\right)\right)$ & {$[0.4,0.7]$} & {$[0.3,0.7]$} & {$[0.3,0.5]$} & {$[0.8,0.9]$} & {$[0.4,0.5]$} & {$[0.2,0.6]$} \\
$\left(e_{2},\left(e_{3}, e_{4}\right)\right)$ & {$[0.5,0.8]$} & {$[0.2,0.6]$} & {$[0.5,0.7]$} & {$[0.6,0.9]$} & {$[0.6,0.7]$} & {$[0.4,0.5]$} \\
$\left(e_{2},\left(e_{3}, e_{5}\right)\right)$ & {$[0.4,0.6]$} & {$[0.3,0.7]$} & {$[0.5,0.7]$} & {$[0.6,0.9]$} & {$[0.6,0.7]$} & {$[0.4,0.5]$} \\
\hline
\end{tabular}

Table 3 Tabular representation of the IVF soft set $\mathfrak{N}=(\tilde{N}, C \times C)$

\begin{tabular}{lllllll}
\hline $\boldsymbol{U}$ & $\boldsymbol{h}_{\mathbf{1}}$ & $\boldsymbol{h}_{\mathbf{2}}$ & $\boldsymbol{h}_{\mathbf{3}}$ & $\boldsymbol{h}_{\mathbf{4}}$ & $\boldsymbol{h}_{\mathbf{5}}$ & $\boldsymbol{h}_{\mathbf{6}}$ \\
\hline$\left(e_{4}, e_{4}\right)$ & {$[0.8,0.9]$} & {$[0.2,0.5]$} & {$[0.2,0.4]$} & {$[0.8,0.9]$} & {$[0.3,0.5]$} & {$[0.3,0.6]$} \\
$\left(e_{4}, e_{5}\right)$ & {$[0.8,0.9]$} & {$[0.3,0.7]$} & {$[0.6,0.8]$} & {$[0.8,0.9]$} & {$[0.7,0.8]$} & {$[0.5,0.7]$} \\
$\left(e_{5}, e_{4}\right)$ & {$[0.8,0.9]$} & {$[0.3,0.7]$} & {$[0.6,0.8]$} & {$[0.8,0.9]$} & {$[0.7,0.8]$} & {$[0.5,0.7]$} \\
$\left(e_{5}, e_{5}\right)$ & {$[0.4,0.6]$} & {$[0.3,0.7]$} & {$[0.6,0.8]$} & {$[0.8,0.9]$} & {$[0.7,0.8]$} & {$[0.5,0.7]$} \\
\hline
\end{tabular}

Table 4 Tabular representation of the IVF soft set $\Re=(\tilde{R},(A \times B) \times(C \times C))$

\begin{tabular}{lllllll}
\hline $\boldsymbol{U}$ & $\boldsymbol{h}_{\mathbf{1}}$ & $\boldsymbol{h}_{\mathbf{2}}$ & $\boldsymbol{h}_{\mathbf{3}}$ & $\boldsymbol{h}_{\mathbf{4}}$ & $\boldsymbol{h}_{\mathbf{5}}$ & $\boldsymbol{h}_{\mathbf{6}}$ \\
\hline$\left(\left(e_{1}, e_{3}\right),\left(e_{4}, e_{4}\right)\right)$ & {$[0.5,0.8]$} & {$[0.2,0.5]$} & {$[0.2,0.4]$} & {$[0.8,0.9]$} & {$[0.3,0.5]$} & {$[0.2,0.6]$} \\
$\left(\left(e_{1}, e_{3}\right),\left(e_{4}, e_{5}\right)\right)$ & {$[0.5,0.8]$} & {$[0.3,0.7]$} & {$[0.3,0.5]$} & {$[0.8,0.9]$} & {$[0.4,0.5]$} & {$[0.2,0.6]$} \\
$\left(\left(e_{1}, e_{3}\right),\left(e_{5}, e_{4}\right)\right)$ & {$[0.5,0.8]$} & {$[0.3,0.7]$} & {$[0.3,0.5]$} & {$[0.8,0.9]$} & {$[0.4,0.5]$} & {$[0.2,0.6]$} \\
$\left(\left(e_{1}, e_{3}\right),\left(e_{5}, e_{5}\right)\right)$ & {$[0.4,0.6]$} & {$[0.3,0.7]$} & {$[0.3,0.5]$} & {$[0.8,0.9]$} & {$[0.4,0.5]$} & {$[0.2,0.6]$} \\
$\left(\left(e_{2}, e_{3}\right),\left(e_{4}, e_{4}\right)\right)$ & {$[0.5,0.8]$} & {$[0.2,0.5]$} & {$[0.2,0.4]$} & {$[0.6,0.9]$} & {$[0.3,0.5]$} & {$[0.3,0.5]$} \\
$\left(\left(e_{2}, e_{3}\right),\left(e_{4}, e_{5}\right)\right)$ & {$[0.5,0.8]$} & {$[0.3,0.7]$} & {$[0.5,0.7]$} & {$[0.6,0.9]$} & {$[0.6,0.7]$} & {$[0.4,0.5]$} \\
$\left(\left(e_{2}, e_{3}\right),\left(e_{5}, e_{4}\right)\right)$ & {$[0.5,0.8]$} & {$[0.3,0.7]$} & {$[0.5,0.7]$} & {$[0.6,0.9]$} & {$[0.6,0.7]$} & {$[0.4,0.5]$} \\
$\left(\left(e_{2}, e_{3}\right),\left(e_{5}, e_{5}\right)\right)$ & {$[0.4,0.6]$} & {$[0.3,0.7]$} & {$[0.5,0.7]$} & {$[0.6,0.9]$} & {$[0.6,0.7]$} & {$[0.4,0.5]$} \\
\hline
\end{tabular}

Moreover, let $\mathfrak{R}=(\widetilde{R},(A \times B) \times(C \times C))=(\widetilde{M}, A \times B) \wedge(\widetilde{N}, C \times C)$, where $\widetilde{R}((a, b)$, $\left.\left(c_{1}, c_{2}\right)\right)=\widetilde{M}(a, b) \cap \widetilde{N}\left(c_{1}, c_{2}\right)=(\widetilde{F}(a) \cup \widetilde{G}(b)) \cap\left(\widetilde{H}\left(c_{1}\right) \cup \widetilde{H}\left(c_{2}\right)\right)$ for all $\left((a, b),\left(c_{1}, c_{2}\right)\right) \in(A \times$ $B) \times(C \times C)$. It is not difficult to check that

$$
\begin{aligned}
(A \times B) \times(C \times C)= & \left\{\left(\left(e_{1}, e_{3}\right),\left(e_{4}, e_{4}\right)\right),\left(\left(e_{1}, e_{3}\right),\left(e_{4}, e_{5}\right)\right),\right. \\
& \left(\left(e_{1}, e_{3}\right),\left(e_{5}, e_{4}\right)\right),\left(\left(e_{1}, e_{3}\right),\left(e_{5}, e_{5}\right)\right), \\
& \left(\left(e_{2}, e_{3}\right),\left(e_{4}, e_{4}\right)\right),\left(\left(e_{2}, e_{3}\right),\left(e_{4}, e_{5}\right)\right), \\
& \left.\left(\left(e_{2}, e_{3}\right),\left(e_{5}, e_{4}\right)\right),\left(\left(e_{2}, e_{3}\right),\left(e_{5}, e_{5}\right)\right)\right\} .
\end{aligned}
$$

Proceeding with detailed calculations, we get the IVF soft set $\mathfrak{R}$ with its tabular representation shown in Table 4.

$$
\text { Also, let } \mathfrak{R}_{1}=\left(\widetilde{R}_{1},(A \times B) \times C\right)=(\widetilde{M}, A \times B) \wedge(\widetilde{H}, C) \text {. Then we have }
$$

$$
(A \times B) \times C=\left\{\left(\left(e_{1}, e_{3}\right), e_{4}\right),\left(\left(e_{1}, e_{3}\right), e_{5}\right),\left(\left(e_{2}, e_{3}\right), e_{4}\right),\left(\left(e_{2}, e_{3}\right), e_{5}\right)\right\},
$$

and tabular representation of the IVF soft set $\Re_{1}$ is shown in Table 5 .

Now, in view of Table 2 and Table 4 one can verify that $\mathfrak{L} \widetilde{\subseteq}_{J} \mathfrak{R}$. That is,

$$
(\widetilde{F}, A) \vee((\widetilde{G}, B) \wedge(\widetilde{H}, C)) \widetilde{\subseteq}_{J}((\widetilde{F}, A) \vee(\widetilde{G}, B)) \wedge((\widetilde{H}, C) \vee(\widetilde{H}, C))
$$

Nevertheless, it is easily seen from Table 2 and Table 5 that $\mathfrak{L} \widetilde{\Phi}_{J} \Re_{1}$ since $\widetilde{L}\left(e_{1},\left(e_{3}, e_{5}\right)\right) \nsubseteq$ $\widetilde{R}\left(\left(e_{1}, e_{3}\right), e_{4}\right), \widetilde{L}\left(e_{1},\left(e_{3}, e_{5}\right)\right) \nsubseteq \widetilde{R}\left(\left(e_{1}, e_{3}\right), e_{5}\right), \widetilde{L}\left(e_{1},\left(e_{3}, e_{5}\right)\right) \nsubseteq \widetilde{R}\left(\left(e_{2}, e_{3}\right), e_{4}\right)$, and $\widetilde{L}\left(e_{1},\left(e_{3}, e_{5}\right)\right) \nsubseteq$ 
Table 5 Tabular representation of the IVF soft set $\Re_{1}=\left(\tilde{R}_{1},(A \times B) \times C\right)$

\begin{tabular}{lllllll}
\hline $\boldsymbol{U}$ & $\boldsymbol{h}_{\mathbf{1}}$ & $\boldsymbol{h}_{\mathbf{2}}$ & $\boldsymbol{h}_{\mathbf{3}}$ & $\boldsymbol{h}_{\mathbf{4}}$ & $\boldsymbol{h}_{\mathbf{5}}$ & $\boldsymbol{h}_{\mathbf{6}}$ \\
\hline$\left(\left(e_{1}, e_{3}\right), e_{4}\right)$ & {$[0.5,0.8]$} & {$[0.2,0.5]$} & {$[0.2,0.4]$} & {$[0.8,0.9]$} & {$[0.3,0.5]$} & {$[0.2,0.6]$} \\
$\left(\left(e_{1}, e_{3}\right), e_{5}\right)$ & {$[0.4,0.6]$} & {$[0.3,0.7]$} & {$[0.3,0.5]$} & {$[0.8,0.9]$} & {$[0.4,0.5]$} & {$[0.2,0.6]$} \\
$\left(\left(e_{2}, e_{3}\right), e_{4}\right)$ & {$[0.5,0.8]$} & {$[0.2,0.5]$} & {$[0.2,0.4]$} & {$[0.6,0.9]$} & {$[0.3,0.5]$} & {$[0.3,0.5]$} \\
$\left(\left(e_{2}, e_{3}\right), e_{5}\right)$ & {$[0.4,0.6]$} & {$[0.3,0.7]$} & {$[0.5,0.7]$} & {$[0.6,0.9]$} & {$[0.6,0.7]$} & {$[0.4,0.5]$} \\
\hline
\end{tabular}

$\widetilde{R}\left(\left(e_{2}, e_{3}\right), e_{5}\right)$. That is,

$$
(\widetilde{F}, A) \vee((\widetilde{G}, B) \wedge(\widetilde{H}, C)) \widetilde{\widetilde{C}}_{J}((\widetilde{F}, A) \vee(\widetilde{G}, B)) \wedge(\widetilde{H}, C)
$$

does not hold in general.

Remark 5.8 In view of Theorem 5.1, Theorem 5.6 and Example 5.7, we can find that under soft product operations, IVF soft sets possess some interesting algebraic properties which differ from those of interval-valued fuzzy sets. In particular, one can see that usual modular inequalities in lattice theory are not valid for IVF soft sets.

Using the generalized commutative laws of IVF soft sets in Theorem 2.9, we have the following consequences of Theorem 5.6.

Corollary 5.9 Let $(\widetilde{F}, A),(\widetilde{G}, B)$, and $(\widetilde{H}, C)$ be IVF soft sets over $U$. If $(\widetilde{F}, A) \widetilde{\subseteq}_{J}(\widetilde{H}, C)$, then we have

(1) $(\widetilde{F}, A) \vee((\widetilde{H}, C) \wedge(\widetilde{G}, B)) \widetilde{\subseteq}_{J}((\widetilde{F}, A) \vee(\widetilde{G}, B)) \wedge((\widetilde{H}, C) \vee(\widetilde{H}, C))$;

(2) $(\widetilde{F}, A) \vee((\widetilde{H}, C) \wedge(\widetilde{G}, B)) \widetilde{\widetilde{C}}_{J}((\widetilde{G}, B) \vee(\widetilde{F}, A)) \wedge((\widetilde{H}, C) \vee(\widetilde{H}, C))$;

(3) $(\widetilde{F}, A) \vee((\widetilde{G}, B) \wedge(\widetilde{H}, C)) \underline{\widetilde{\subseteq}}_{J}((\widetilde{G}, B) \vee(\widetilde{F}, A)) \wedge((\widetilde{H}, C) \vee(\widetilde{H}, C))$.

Proof We only verify the first assertion; the proofs of the others can be obtained in a similar fashion. First, by the generalized commutative laws of IVF soft sets, we also know that

$$
(\widetilde{G}, B) \wedge(\widetilde{H}, C)=_{L}(\widetilde{H}, C) \wedge(\widetilde{G}, B)
$$

and so

$$
(\widetilde{H}, C) \wedge(\widetilde{G}, B) \widetilde{\subseteq}_{J}(\widetilde{G}, B) \wedge(\widetilde{H}, C) .
$$

Now, from Proposition 3.6, it follows that

$$
(\widetilde{F}, A) \vee((\widetilde{H}, C) \wedge(\widetilde{G}, B)) \widetilde{\subseteq}_{J}(\widetilde{F}, A) \vee((\widetilde{G}, B) \wedge(\widetilde{H}, C))
$$

But according to Theorem 5.6,

$$
(\widetilde{F}, A) \vee((\widetilde{G}, B) \wedge(\widetilde{H}, C)) \widetilde{\subseteq}_{J}((\widetilde{F}, A) \vee(\widetilde{G}, B)) \wedge((\widetilde{H}, C) \vee(\widetilde{H}, C))
$$

Hence we conclude that

$$
(\widetilde{F}, A) \vee((\widetilde{H}, C) \wedge(\widetilde{G}, B)) \widetilde{\subseteq}_{J}((\widetilde{F}, A) \vee(\widetilde{G}, B)) \wedge((\widetilde{H}, C) \vee(\widetilde{H}, C))
$$

by transitivity of the preorder $\widetilde{\subseteq}_{J}$ on $\mathscr{S}^{I}(U, E)$. 
Using similar techniques as above, we obtain the following results.

Corollary 5.10 Let $(\widetilde{F}, A),(\widetilde{G}, B)$, and $(\widetilde{H}, C)$ be IVF soft sets over $U$. If $(\widetilde{F}, A) \widetilde{\subseteq}_{J}(\widetilde{H}, C)$, then we have

(1) $(\widetilde{F}, A) \vee((\widetilde{H}, C) \wedge(\widetilde{G}, B)) \widetilde{\subseteq}_{J}((\widetilde{H}, C) \vee(\widetilde{H}, C)) \wedge((\widetilde{G}, B) \vee(\widetilde{F}, A))$;

(2) $(\widetilde{F}, A) \vee((\widetilde{G}, B) \wedge(\widetilde{H}, C)) \widetilde{\subseteq}_{J}((\widetilde{H}, C) \vee(\widetilde{H}, C)) \wedge((\widetilde{G}, B) \vee(\widetilde{F}, A))$;

(3) $(\widetilde{F}, A) \vee((\widetilde{G}, B) \wedge(\widetilde{H}, C)) \widetilde{\widetilde{C}}_{J}((\widetilde{H}, C) \vee(\widetilde{H}, C)) \wedge((\widetilde{F}, A) \vee(\widetilde{G}, B))$;

(4) $(\widetilde{F}, A) \vee((\widetilde{H}, C) \wedge(\widetilde{G}, B)) \underline{\widetilde{\subseteq}}_{J}((\widetilde{H}, C) \vee(\widetilde{H}, C)) \wedge((\widetilde{F}, A) \vee(\widetilde{G}, B))$.

Corollary 5.11 Let $(\widetilde{F}, A),(\widetilde{G}, B)$, and $(\widetilde{H}, C)$ be IVF soft sets over $U$. If $(\widetilde{F}, A) \widetilde{\subseteq}_{J}(\widetilde{H}, C)$, then we have

(1) $((\widetilde{H}, C) \wedge(\widetilde{G}, B)) \vee(\widetilde{F}, A) \widetilde{\widetilde{\subseteq}}_{J}((\widetilde{H}, C) \vee(\widetilde{H}, C)) \wedge((\widetilde{G}, B) \vee(\widetilde{F}, A))$;

(2) $((\widetilde{G}, B) \wedge(\widetilde{H}, C)) \vee(\widetilde{F}, A) \underline{\widetilde{\subseteq}}_{J}((\widetilde{H}, C) \vee(\widetilde{H}, C)) \wedge((\widetilde{G}, B) \vee(\widetilde{F}, A))$;

(3) $((\widetilde{G}, B) \wedge(\widetilde{H}, C)) \vee(\widetilde{F}, A) \widetilde{\subseteq}_{J}((\widetilde{H}, C) \vee(\widetilde{H}, C)) \wedge((\widetilde{F}, A) \vee(\widetilde{G}, B))$;

(4) $((\widetilde{H}, C) \wedge(\widetilde{G}, B)) \vee(\widetilde{F}, A) \widetilde{\subseteq}_{J}((\widetilde{H}, C) \vee(\widetilde{H}, C)) \wedge((\widetilde{F}, A) \vee(\widetilde{G}, B))$.

Corollary 5.12 Let $(\widetilde{F}, A),(\widetilde{G}, B)$, and $(\widetilde{H}, C)$ be IVF soft sets over $U$. If $(\widetilde{F}, A) \widetilde{\subseteq}_{J}(\widetilde{H}, C)$, then we have

(1) $((\widetilde{H}, C) \wedge(\widetilde{G}, B)) \vee(\widetilde{F}, A) \widetilde{\subseteq}_{J}((\widetilde{G}, B) \vee(\widetilde{F}, A)) \wedge((\widetilde{H}, C) \vee(\widetilde{H}, C))$;

(2) $((\widetilde{G}, B) \wedge(\widetilde{H}, C)) \vee(\widetilde{F}, A) \widetilde{\subseteq}_{J}((\widetilde{G}, B) \vee(\widetilde{F}, A)) \wedge((\widetilde{H}, C) \vee(\widetilde{H}, C))$;

(3) $((\widetilde{G}, B) \wedge(\widetilde{H}, C)) \vee(\widetilde{F}, A) \widetilde{\subseteq}_{J}((\widetilde{F}, A) \vee(\widetilde{G}, B)) \wedge((\widetilde{H}, C) \vee(\widetilde{H}, C))$;

(4) $((\widetilde{H}, C) \wedge(\widetilde{G}, B)) \vee(\widetilde{F}, A) \widetilde{\subseteq}_{J}((\widetilde{F}, A) \vee(\widetilde{G}, B)) \wedge((\widetilde{H}, C) \vee(\widetilde{H}, C))$.

Theorem 5.13 Let $(\widetilde{F}, A),(\widetilde{G}, B)$, and $(\widetilde{H}, C)$ be IVF soft sets over $U$.If $(\widetilde{F}, A) \widetilde{\subseteq}_{J}(\widetilde{H}, C)$ and $(\widetilde{H}, C)$ is upward directed, then we have

$$
(\widetilde{F}, A) \vee((\widetilde{G}, B) \wedge(\widetilde{H}, C))=_{J}((\widetilde{F}, A) \vee(\widetilde{G}, B)) \wedge(\widetilde{H}, C)
$$

Proof First, according to Theorem 5.6,

$$
(\widetilde{F}, A) \vee((\widetilde{G}, B) \wedge(\widetilde{H}, C)) \widetilde{\subseteq}_{J}((\widetilde{F}, A) \vee(\widetilde{G}, B)) \wedge((\widetilde{H}, C) \vee(\widetilde{H}, C)) .
$$

But by the hypothesis, $(\widetilde{H}, C)$ is a upward directed IVF soft set, and so

$$
(\widetilde{H}, C) \vee(\widetilde{H}, C)=_{J}(\widetilde{H}, C)
$$

by Proposition 4.7. It follows that

$$
(\widetilde{H}, C) \vee(\widetilde{H}, C) \widetilde{\subseteq}_{J}(\widetilde{H}, C)
$$

and so by Proposition 3.10, we deduce that

$$
((\widetilde{F}, A) \vee(\widetilde{G}, B)) \wedge((\widetilde{H}, C) \vee(\widetilde{H}, C)) \widetilde{\subseteq}_{J}((\widetilde{F}, A) \vee(\widetilde{G}, B)) \wedge(\widetilde{H}, C)
$$

Thus by transitivity of $\widetilde{\subseteq}_{J}$, we have

$$
(\widetilde{F}, A) \vee((\widetilde{G}, B) \wedge(\widetilde{H}, C)) \widetilde{\subseteq}_{J}((\widetilde{F}, A) \vee(\widetilde{G}, B)) \wedge(\widetilde{H}, C) .
$$


On the other hand, we also have

$$
((\widetilde{F}, A) \vee(\widetilde{G}, B)) \wedge(\widetilde{H}, C) \widetilde{\subseteq}_{J}(\widetilde{F}, A) \vee((\widetilde{G}, B) \wedge(\widetilde{H}, C))
$$

according to Theorem 5.1. Therefore, we finally conclude that

$$
(\widetilde{F}, A) \vee((\widetilde{G}, B) \wedge(\widetilde{H}, C))=_{J}((\widetilde{F}, A) \vee(\widetilde{G}, B)) \wedge(\widetilde{H}, C),
$$

completing the proof.

Using the generalized commutative laws of IVF soft sets in Theorem 2.9, we have the following consequences of Theorem 5.13.

Corollary 5.14 Let $(\widetilde{F}, A),(\widetilde{G}, B)$, and $(\widetilde{H}, C)$ be IVF soft sets over $U$. If $(\widetilde{F}, A) \widetilde{\subseteq}_{J}(\widetilde{H}, C)$ and $(\widetilde{H}, C)$ is upward directed, then we have

(1) $(\widetilde{F}, A) \vee((\widetilde{H}, C) \wedge(\widetilde{G}, B))={ }_{J}((\widetilde{F}, A) \vee(\widetilde{G}, B)) \wedge(\widetilde{H}, C)$;

(2) $(\widetilde{F}, A) \vee((\widetilde{H}, C) \wedge(\widetilde{G}, B))=_{J}((\widetilde{G}, B) \vee(\widetilde{F}, A)) \wedge(\widetilde{H}, C)$;

(3) $(\widetilde{F}, A) \vee((\widetilde{G}, B) \wedge(\widetilde{H}, C))={ }_{J}((\widetilde{G}, B) \vee(\widetilde{F}, A)) \wedge(\widetilde{H}, C)$.

Proof By the generalized commutative laws of IVF soft sets, we have

$$
(\widetilde{G}, B) \wedge(\widetilde{H}, C)=_{L}(\widetilde{H}, C) \wedge(\widetilde{G}, B)
$$

and so

$$
(\widetilde{G}, B) \wedge(\widetilde{H}, C)=_{J}(\widetilde{H}, C) \wedge(\widetilde{G}, B)
$$

That is,

$$
(\widetilde{G}, B) \wedge(\widetilde{H}, C) \widetilde{\subseteq}_{J}(\widetilde{H}, C) \wedge(\widetilde{G}, B)
$$

and

$$
(\widetilde{H}, C) \wedge(\widetilde{G}, B) \widetilde{\subseteq}_{J}(\widetilde{G}, B) \wedge(\widetilde{H}, C)
$$

Then according to Proposition 3.10, we deduce that

$$
(\widetilde{F}, A) \vee((\widetilde{G}, B) \wedge(\widetilde{H}, C)) \widetilde{\subseteq}_{J}(\widetilde{F}, A) \vee((\widetilde{H}, C) \wedge(\widetilde{G}, B))
$$

and

$$
(\widetilde{F}, A) \vee((\widetilde{H}, C) \wedge(\widetilde{G}, B)) \widetilde{\subseteq}_{J}(\widetilde{F}, A) \vee((\widetilde{G}, B) \wedge(\widetilde{H}, C))
$$

Hence it follows that

$$
(\widetilde{F}, A) \vee((\widetilde{H}, C) \wedge(\widetilde{G}, B))=_{J}(\widetilde{F}, A) \vee((\widetilde{G}, B) \wedge(\widetilde{H}, C))
$$


Now, according to Theorem 5.13,

$$
(\widetilde{F}, A) \vee((\widetilde{G}, B) \wedge(\widetilde{H}, C))=_{J}((\widetilde{F}, A) \vee(\widetilde{G}, B)) \wedge(\widetilde{H}, C)
$$

Therefore, we conclude that

$$
(\widetilde{F}, A) \vee((\widetilde{H}, C) \wedge(\widetilde{G}, B))=_{J}((\widetilde{F}, A) \vee(\widetilde{G}, B)) \wedge(\widetilde{H}, C)
$$

by transitivity of IVF soft $J$-equal relations. The proofs of other soft $J$-equalities can be obtained using similar techniques.

The proofs of the following results are similar to that of Corollary 5.14 and thus omitted.

Corollary 5.15 Let $(\widetilde{F}, A),(\widetilde{G}, B)$, and $(\widetilde{H}, C)$ be IVF soft sets over $U$.If $(\widetilde{F}, A) \widetilde{\subseteq}_{J}(\widetilde{H}, C)$ and $(\widetilde{H}, C)$ is upward directed, then we have

(1) $((\widetilde{H}, C) \wedge(\widetilde{G}, B)) \vee(\widetilde{F}, A)=_{J}((\widetilde{F}, A) \vee(\widetilde{G}, B)) \wedge(\widetilde{H}, C)$;

(2) $((\widetilde{H}, C) \wedge(\widetilde{G}, B)) \vee(\widetilde{F}, A)=_{J}((\widetilde{G}, B) \vee(\widetilde{F}, A)) \wedge(\widetilde{H}, C)$;

(3) $((\widetilde{G}, B) \wedge(\widetilde{H}, C)) \vee(\widetilde{F}, A)=_{J}((\widetilde{G}, B) \vee(\widetilde{F}, A)) \wedge(\widetilde{H}, C)$;

(4) $((\widetilde{G}, B) \wedge(\widetilde{H}, C)) \vee(\widetilde{F}, A)=_{J}((\widetilde{F}, A) \vee(\widetilde{G}, B)) \wedge(\widetilde{H}, C)$.

Corollary 5.16 Let $(\widetilde{F}, A),(\widetilde{G}, B)$, and $(\widetilde{H}, C)$ be IVF soft sets over $U$.If $(\widetilde{F}, A) \widetilde{\subseteq}_{J}(\widetilde{H}, C)$ and $(\widetilde{H}, C)$ is upward directed, then we have

(1) $((\widetilde{H}, C) \wedge(\widetilde{G}, B)) \vee(\widetilde{F}, A)=_{J}(\widetilde{H}, C) \wedge((\widetilde{F}, A) \vee(\widetilde{G}, B))$;

(2) $((\widetilde{H}, C) \wedge(\widetilde{G}, B)) \vee(\widetilde{F}, A)=_{J}(\widetilde{H}, C) \wedge((\widetilde{G}, B) \vee(\widetilde{F}, A))$;

(3) $((\widetilde{G}, B) \wedge(\widetilde{H}, C)) \vee(\widetilde{F}, A)=_{J}(\widetilde{H}, C) \wedge((\widetilde{G}, B) \vee(\widetilde{F}, A))$;

(4) $((\widetilde{G}, B) \wedge(\widetilde{H}, C)) \vee(\widetilde{F}, A)=_{J}(\widetilde{H}, C) \wedge((\widetilde{F}, A) \vee(\widetilde{G}, B))$.

Corollary 5.17 Let $(\widetilde{F}, A),(\widetilde{G}, B)$, and $(\widetilde{H}, C)$ be IVF soft sets over $U$.If $(\widetilde{F}, A) \widetilde{\subseteq}_{J}(\widetilde{H}, C)$ and $(\widetilde{H}, C)$ is upward directed, then we have

(1) $(\widetilde{F}, A) \vee((\widetilde{H}, C) \wedge(\widetilde{G}, B))=_{J}(\widetilde{H}, C) \wedge((\widetilde{F}, A) \vee(\widetilde{G}, B))$;

(2) $(\widetilde{F}, A) \vee((\widetilde{H}, C) \wedge(\widetilde{G}, B))=_{J}(\widetilde{H}, C) \wedge((\widetilde{G}, B) \vee(\widetilde{F}, A))$;

(3) $(\widetilde{F}, A) \vee((\widetilde{G}, B) \wedge(\widetilde{H}, C))=_{J}(\widetilde{H}, C) \wedge((\widetilde{G}, B) \vee(\widetilde{F}, A))$;

(4) $(\widetilde{F}, A) \vee((\widetilde{G}, B) \wedge(\widetilde{H}, C))=_{J}(\widetilde{H}, C) \wedge((\widetilde{F}, A) \vee(\widetilde{G}, B))$.

\section{Conclusions}

This paper focused on exploring modular inequalities of IVF soft sets characterized by Jun's soft $J$-inclusions. It has been shown that Jun's soft $J$-inclusions and Liu's soft $L$ inclusions are preorders; hence the soft equal relations $=_{J}$ and $=_{L}$ derived from them are equivalence relations on the collection $\mathscr{S}^{I}(U, E)$ of all IVF soft sets over $U$ with parameter space $E$. These soft inclusions proved to be useful in characterizing some fundamental algebraic properties of soft product operations. Moreover, upward directed IVF soft sets were introduced and some equivalent characterizations were presented. We finally considered modular laws in lattice theory and proposed modular inequalities of IVF soft sets using soft $J$-inclusions and some related notions mentioned above. As future work, we will further investigate other types of interesting inequalities of IVF soft sets. 


\section{Competing interests}

The authors declare that they have no competing interests.

\section{Authors' contributions}

All authors read and approved the final manuscript.

\section{Author details}

'Department of Applied Mathematics, School of Science, Xi'an University of Posts and Telecommunications, Xi'an, 710121, China. ${ }^{2}$ Machine Intelligence Institute, lona College, New Rochelle, 10801, USA. ${ }^{3}$ Department of Mathematics, Yazd University, Yazd, Iran. ${ }^{4}$ Department of Mathematics, COMSATS Institute of Information Technology, Abbottabad, 22060, Pakistan. ${ }^{5}$ Department of Mathematics, University of Chicago, Chicago, 60637, USA.

\section{Acknowledgements}

The authors are highly grateful to the anonymous referees for their insightful comments and valuable suggestions which greatly improve the quality of this paper. This work was partially supported by National Natural Science Foundation of China (Program No. 11301415), Natural Science Basic Research Plan in Shaanxi Province of China (Program No. 2013JQ1020), Shaanxi Provincial Research Plan for Young Scientific and Technological New Stars (Program No. 2014KJXX-73) and Scientific Research Program Funded by Shaanxi Provincial Education Department of China (Program No. 2013JK1098).

Received: 21 February 2014 Accepted: 15 September 2014 Published: 24 Sep 2014

\section{References}

1. Zadeh, LA: Fuzzy sets. Inf. Control 8, 338-353 (1965)

2. Pawlak, Z: Rough sets. Int. J. Comput. Inf. Sci. 11, 341-356 (1982)

3. Molodtsov, DA: Soft set theory-first results. Comput. Math. Appl. 37, 19-31 (1999)

4. Aktaş, H, Çağman, N: Soft sets and soft groups. Inf. Sci. 177, 2726-2735 (2007)

5. Jun, YB, Park, CH: Applications of soft sets in ideal theory of BCK/BCl-algebras. Inf. Sci. 178, 2466-2475 (2008)

6. Jun, YB, Lee, KJ, Zhan, J: Soft p-ideals of soft BCl-algebras. Comput. Math. Appl. 58, 2060-2068 (2009)

7. Jun, YB, Lee, KJ, Khan, A: Soft ordered semigroups. Math. Log. Q. 56, $42-50$ (2010)

8. Jun, YB, Song, SZ, So, KS: Soft set theory applied to $p$-ideals of $\mathrm{BCl}$-algebras related to fuzzy points. Neural Comput. Appl. 20, 1313-1320 (2011)

9. Atagün, AO, Sezgin, A: Soft substructures of rings, fields and modules. Comput. Math. Appl. 61, $592-601$ (2011)

10. Akram, M, Alshehri, NO, Alghamdi, RS: Fuzzy soft K-algebras. Util. Math. 90, 307-325 (2013)

11. Akram, M: Bipolar fuzzy soft Lie algebras. Quasigr. Relat. Syst. 21, 1-10 (2013)

12. Yaqoob, N, Akram, M, Aslam, M: Intuitionistic fuzzy soft groups induced by $(t, s)$-norm. Indian J. Inf. Sci. Technol. 6 , 4282-4289 (2013)

13. Shabir, M, Naz, M: On soft topological spaces. Comput. Math. Appl. 61, 1786-1799 (2011)

14. Zou, Y, Xiao, Z: Data analysis approaches of soft sets under incomplete information. Knowl.-Based Syst. 21, 941-945 (2008)

15. Çağman, N, Enginoğlu, S: Soft set theory and uni-int decision making. Eur. J. Oper. Res. 207, 848-855 (2010)

16. Qin, KY, Hong, ZY: On soft equality. J. Comput. Appl. Math. 234, 1347-1355 (2010)

17. Gong, K, Xiao, Z, Zhang, X: Exclusive disjunctive soft sets. Comput. Math. Appl. 60, 2270-2278 (2010)

18. Ali, MI, Feng, F, Liu, XY, Min, WK, Shabir, M: On some new operations in soft set theory. Comput. Math. Appl. 57, 1547-1553 (2009)

19. Ali, MI, Shabir, M, Naz, M: Algebraic structures of soft sets associated with new operations. Comput. Math. Appl. 61 , 2647-2654 (2011)

20. Ali, Ml: A note on soft sets, rough soft sets and fuzzy soft sets. Appl. Soft Comput. 11, 3329-3332 (2011)

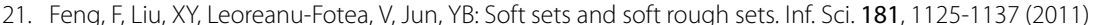

22. Feng, F, Li, CX, Davvaz, B, Irfan Ali, M: Soft sets combined with fuzzy sets and rough sets: a tentative approach. Soft Comput. 14, 899-911 (2010)

23. Maji, PK, Biswas, R, Roy, AR: Soft set theory. Comput. Math. Appl. 45, 555-562 (2003)

24. Majumdar, P, Samanta, SK: Generalised fuzzy soft sets. Comput. Math. Appl. 59, 1425-1432 (2010)

25. Maji, PK, Biswas, R, Roy, AR: Fuzzy soft sets. J. Fuzzy Math. 9, 589-602 (2001)

26. Yang, $X B$, Lin, TY, Yang, JY, Li, Y, Yu, DJ: Combination of interval-valued fuzzy set and soft set. Comput. Math. Appl. 58 521-527 (2009)

27. Jun, YB, Yang, X: A note on the paper 'Combination of interval-valued fuzzy set and soft set' [Comput. Math. Appl. 58 (2009) 521-527]. Comput. Math. Appl. 61, 1468-1470 (2011)

28. Liu, XY, Feng, F, Jun, YB: A note on generalized soft equal relations. Comput. Math. Appl. 64, 572-578 (2012)

29. Feng, F, Li, YM: Soft subsets and soft product operations. Inf. Sci. 232, 44-57 (2013)

30. Liu, XY, Feng, F, Zhang, H: On some nonclassical algebraic properties of interval-valued fuzzy soft sets. Sci. World J. 2014, Article ID 192957 (2014)

31. Feng, F, Li, YM, Leoreanu-Fotea, V: Application of level soft sets in decision making based on interval-valued fuzzy soft sets. Comput. Math. Appl. 60, 1756-1767 (2010)

10.1186/1029-242X-2014-360

Cite this article as: Liu et al.: On modular inequalities of interval-valued fuzzy soft sets characterized by soft

J-inclusions. Journal of Inequalities and Applications 2014, 2014:360 